\title{
FMZ-PET to assess the efficacy and the mechanism of ketogenic diet in patients with intractable epilepsy
}

Objective: A ketogenic diet (KD) is a strict dietary protocol that incorporates high-fat, low-protein, and low-carbohydrate foods. Increasing the levels of ketones became an eventual and effective treatment strategy for medically refractory epilepsy. Although several theories on the rationale for the anticonvulsant efficacy of KD have been hypothesized, the most important anticonvulsant mechanism of KD is still unknown. This study is the first report that evaluated the mechanism underlying the effect of KD in patients with intractable infantile epilepsy using FMZ-PET imaging studies.

Methods: Six patients with intractable epilepsy (two females/four males; seizure-onset age: 0-30 months) were enrolled in this study. Medication-refractory seizures continued in all patients despite treatment with the anti-epilepsy drugs (AEDs), followed by two types of KD therapy; the classical KD menu or the MAD regimen. All patients underwent FMZ-PET before KD and at 1-5 months after induction of KD. Dynamic FMZ-PET scanning was acquired, followed by assessment of the BP images using the graphic plot method of Logan.

Results: In KD-effective patients, the BPs of FMZ before KD were 1.31, 1.60 and 1.50 and those after induction of KD were $1.89,2.13$ and 2.07, respectively. In KD-partially effective patients, the BPs of FMZ before KD was 1.95 and 2.47 and those after induction of KD were 2.10 and 2.71, respectively. Conversely, in KD-ineffective case, the BP of FMZ was decreased from 3.91 before KD to 3.58. The BPchange ratios were $1.44,1.33$ and 1.38 in the KD-effective patients, 1.08 and 1.10 in the KD-partially effective patients, and 0.92 in the KD-ineffective one.

Conclusion: The results of current study suggested that KD may control seizures by increasing BP of BZR and that FMZ-PET imaging would be useful to assess the efficacy of KD therapy in patients with intractable epilepsy.

\section{KEYWORDS: flumazenil-PET - GABA receptor - binding potential - ketogenic diet - intractable epilepsy}

\section{Introduction}

A ketogenic diet $(\mathrm{KD})$ is a strict dietary protocol that incorporates high-fat, low-protein, and low-carbohydrate foods and has been used since the 1920s. In KD, the energy source for the body comes from using fats instead of glucose; this process is called ketosis. For some patients with epilepsy, seizures were prevented when the body made ketones [1,2]. Therefore, increasing the levels of ketones became an eventual and effective treatment strategy for medically refractory epilepsy [1,2]. On administering $\mathrm{KD}$ therapy, approximately $50 \%$ of children who failed on medications had a greater than $50 \%$ reduction in seizures, and approximately $10 \%$ of children who failed on medications became completely seizure- and medicationfree [2]. Since the development of the modified Atkins diet (MAD) at Johns Hopkins Hospital as a more palatable and less restrictive dietary treatment for intractable epilepsy than classical $\mathrm{KD}$ [3], reports of successful use of MAD in children with intractable epilepsy have been published [4-10].

For the proposed mechanism of the KD effects, several hypotheses have been investigated [11-14]: (a) the production of ketones, (b) the glucose restriction, (c) the elevation of free fatty acids, or (d) the metabolic modification of neurotransmitter synthesis and release. Specifically, ketosis would modify the tricarboxylic acid (TCA) cycle and increase glutamate levels and $\gamma$-Aminobutyric acid [15] synthesis, which would then enhance GABAergic output. However, no mechanism has been widely accepted so far because these concepts have been difficult to clarify in in vivo neuroscience studies. Although several theories on the rationale for the anticonvulsant efficacy of KD have been hypothesized, the most important anticonvulsant mechanism of $\mathrm{KD}$ is still unknown.

Molecular imaging by positron emission tomography [16] with ${ }^{11} \mathrm{C}$-flumazenil (FMZ) has been useful in the assessment of patients having neural damage, including epilepsy, because FMZ binds to the benzodiazepine site of the GABA receptor complex in humans [1720]. We also have investigated neural damage in patients with cerebrovascular diseases using the specific parameter of the binding potential (BP) of FMZ to the GABA $A_{A}$ receptor complex in the human brain [21-24].

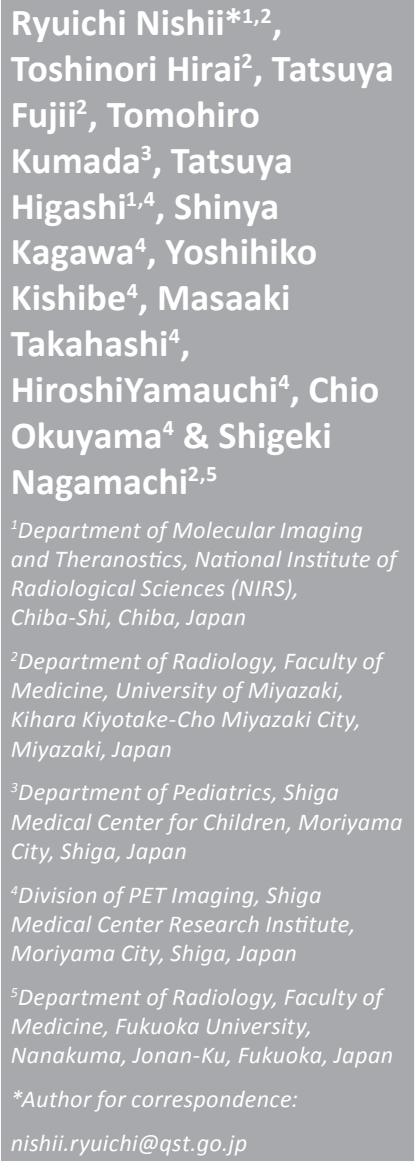


This study is the first report that evaluated the mechanism underlying the effect of KD in patients with intractable infantile epilepsy and that focused on the changes of the binding potentials (BP) of a specific ligand FMZ to benzodiazepine receptor (BZR)- related $\mathrm{GABA}_{\mathrm{A}}-\mathrm{R}$ functions using on FMZ-PET studies.

\section{Materials and Methods - Patients}

We had precious experiences with six patients with intractable epilepsy (two females/four males; seizure-onset age: 0-30 months) who were enrolled in this study. There were tonic spasms in five patients and non-convulsive status epilepticus and tonic and hyper-motor seizures in one patient. Medication-refractory seizures continued in all patients despite treatment with the anti-epilepsy drugs (AEDs), such as sodium valproate (VPA), clobazam (CLB), lamotrigine (LTG), phenobarbital [25] or topiramate (TPM), followed by KD therapy introduced after enough understanding about this diet therapy and agreement of their parents. Two patients began the classical KD regimen and four began the MAD protocol. The clinical diagnoses were performed by pediatric neurologists using clinical manifestations, such as the style and frequency of seizures and the findings on electroencephalogram. Within 1-5 months after KD therapy induction, three patients had improved and two had partially improved; however, the KD therapy was ineffective in one patient because the seizures continued and the abnormal EEG spike and waves were still present. In reference to case \#3, we reported the efficacy of KD in 2012 [26].

The institutional review board approved the protocol of this prospective investigation. All enrolled patients/their parents received thorough explanations and displayed sufficient understanding; they then provided written informed consent regarding this study.

\section{- KD therapy protocol}

The two types of KD protocol - the classical $\mathrm{KD}$ menu or the MAD regimen - were the therapies implemented in this study, as had been described in previous reports $[1,27,28]$. Two dietitians were responsible for all contacts with the families throughout the hospitalization. Before admission, the dietitians estimated the caloric requirements of each patient and identified their favorite foods by interviewing the parents.

For classical KD, the typical ratio of fats to carbohydrates and protein (in terms of grams) was 3:1. For the MAD menu, the carbohydrate content of the diet was restricted to $10 \mathrm{~g} /$ day at the initiation of the diet therapy, and the total calorie content was adjusted to the routine caloric intake. If the patients felt hungry, we allowed an increase of the total daily calories in the diet without exceeding the carbohydrate restriction of $10 \mathrm{~g}$ daily. Fiber was included in the $10 \mathrm{~g}$ of carbohydrates. In the diet, there were no restrictions for the intake of fluids and proteins and no changes in the AED regimen.

\section{- PET imaging and data analysis}

All patients underwent FMZ-PET before $\mathrm{KD}$ and at 1-5 months after induction of KD. PET scans were performed using a whole-body Advance scanner (General Electric Medical System, Milwaukee, Wisconsin), which permitted simultaneous acquisition of 35 image slices with an inter-slice spacing of $4.25 \mathrm{~mm}$ [29]. A transmission scan was performed using $68 \mathrm{Ge} / 68 \mathrm{Ga}$ for attenuation correction in each subject before tracer administration. When reconstructing the PET data using filtered back projection, the images were blurred to a $6.0 \mathrm{~mm}$ full width at half maximum in the trans-axial direction using a Hanning filter. ${ }^{11} \mathrm{C}$-flumazenil (FMZ) was used according to the previously described standard procedure $[24,29,30]$. The FMZ was synthesized in house by ${ }^{11} \mathrm{C}$-methylation of demethylated-flumazenil (Hoffmann-La Roche, Basel, Switzerland). Specific activities at the time of injection ranged from 16.2 to 20.2 (mean 17.8) $\mathrm{GBq} / \mathrm{mmol}$. During PET imaging, all patients were sedated with an intravenous injection of thiopental sodium $(5 \mathrm{~mL} / \mathrm{kg}$ ) while monitoring vital signs. After the slow intravenous injection of 75-130 $\mathrm{MBq}$ of FMZ into a peripheral vein using an automatic injector over $60 \mathrm{~s}$, a $50 \mathrm{~min}$ dynamic PET scan was initiated at the same time as the tracer administration with frame durations of $10 s \times 6,15 s \times 8,30 s \times 4,60 s \times 5,5 \mathrm{~min} \times 4$ and $10 \mathrm{~min} \times 2$.

All PET images were fused with the magnetic resonance (MR) images of each subject using the PMOD software ver. 3.1 (PMOD; 
Zürich, Switzerland) for the interpretation and calculation of the binding potential (BP) of FMZ and for the graphical analysis of the non-invasive parametric image. The BP of FMZ was calculated using dynamic data and a Logan graphical analysis of reference tissue in which the pons was used as the reference region $[29,31,32]$. We selected two tomographic planes that corresponded to the pons from the average-tissue activity image that had been obtained from the early phase of the dynamic PET data with periods of 1-3 min. We manually placed these two planes on an irregular region of interest (ROI) that included almost all pixels that had activity ranging from the maximum pixel value to $50 \%$ of the maximum value. This method had been validated using the data from normal volunteers after comparison with the pons identification on a co-registered MRI [29]; however, it is ideal to place the ROIs on the coregistered MRI images in each patient. These ROIs were transferred to the dynamic PET data for the creation of the parametric images in FMZ BP. The PET images were interpreted and visually assessed by two experienced nuclear medicine physicians who had access to all available clinical information. We compared the relationship of the clinical outcome of each patient with the values of PET before and after the $\mathrm{KD}$ treatment. Changes in the mean value of BP in the cerebral cortex in each patient were also analyzed.

\section{Results}

As summarized in TABLE 1, the BPs of FMZ in the cerebral cortex before $\mathrm{KD}$ were 1.31 ,
1.60 and 1.50 and those after induction of $\mathrm{KD}$ were $1.89,2.13$ and 2.07 in patients in whom seizures disappeared and EEGs improved; as KD-effective, case \#1-3. In the two patients whose seizures were persistent or were reduced in frequency and whose EEGs were partially improved, the BPs of FMZ in the cerebral cortex before KD were 1.95 and 2.47 and those after the induction of KD were 2.10 and 2.71, respectively; as $\mathrm{KD}$-partially effective, case \#4 and 5 . In contrast, the 26 month old male (case \#6) did not respond despite the induction of MAD; as KD-ineffective. His BP of FMZ in the cerebral cortex had decreased from 3.91 before $\mathrm{KD}$ to 3.58 two months after the $\mathrm{KD}$ initiation. The changes of BP of FMZ during $\mathrm{KD}$ are shown in FIGURE 1. The ratios for the BP-changes were $1.44,1.33$, and 1.38 in the KD-effective patients, 1.08 and 1.10 in the KDpartially effective patients, and 0.92 in the KDineffective one (TABLE 2).

FIGURE 2 shows a representative case of a KD-effective patient (case \#1) who had experienced refractory epilepsy while being treated with several AEDs (VPA, CLB, and LTG). The seizures of this patient had disappeared 4 days after the initiation of the MAD protocol. The BP of FMZ was increased and there was no abnormal BP region in the parametric image at one month after KD. FIGURE 3 shows a case of a KD-partially effective patient (case \#4). His seizures were partially improved but continued after one month of classic KD. The hypsarrhythmia that was found on EEG before KD had partially improved to a modified hypsarrhythmia after KD. The BP of FMZ in the cerebral cortex showed no change, and

\begin{tabular}{|c|c|c|c|c|c|c|c|c|c|}
\hline$\#$ & Sex & Age at KD & $\begin{array}{l}\text { Sort of } \\
\text { KD }\end{array}$ & Outcome & $\begin{array}{l}\text { Change } \\
\text { of EEG }\end{array}$ & Seizure & $\begin{array}{l}B P \text { of } F M Z \\
\text { before } K D\end{array}$ & BP of FMZ after KD & $\begin{array}{l}\text { Change } \\
\text { ratio of } B P\end{array}$ \\
\hline 1 & Male & $\begin{array}{l}1 \text { year } 10 \\
\text { months }\end{array}$ & MAD & Effective & Improved & Disappeared & 1.31 & 1.89 (1 month after KD) & 1.44 \\
\hline 2 & Female & 1 year 4 months & MAD & Effective & Improved & Disappeared & 1.60 & 2.13 (1 month after KD) & 1.33 \\
\hline 3 & Female & $\begin{array}{l}4 \text { years } 11 \\
\text { months }\end{array}$ & MAD & Effective & Improved & Disappeared & 1.50 & 2.07 (1 month after KD) & 1.38 \\
\hline 4 & Male & 1 year 2 months & $\begin{array}{c}\text { Classical } \\
\text { KD }\end{array}$ & $\begin{array}{l}\text { Partially } \\
\text { effective }\end{array}$ & $\begin{array}{l}\text { Partially } \\
\text { improved }\end{array}$ & Continued & 1.95 & 2.10 (1 month after KD) & 1.08 \\
\hline 5 & Male & $\begin{array}{l}2 \text { years } 8 \\
\text { months }\end{array}$ & $\begin{array}{l}\text { Classical } \\
\text { KD }\end{array}$ & $\begin{array}{l}\text { Partially } \\
\text { effective }\end{array}$ & $\begin{array}{l}\text { Partially } \\
\text { improved }\end{array}$ & Reduced & 2.47 & 2.71 (1 month after KD) & 1.10 \\
\hline 6 & Male & $\begin{array}{l}2 \text { years } 2 \\
\text { months }\end{array}$ & MAD & Ineffective & $\begin{array}{c}\text { Not } \\
\text { improved }\end{array}$ & Continued & 3.91 & 3.58 (1 month after KD) & 0.92 \\
\hline
\end{tabular}


Table 2. Patient characteristics.

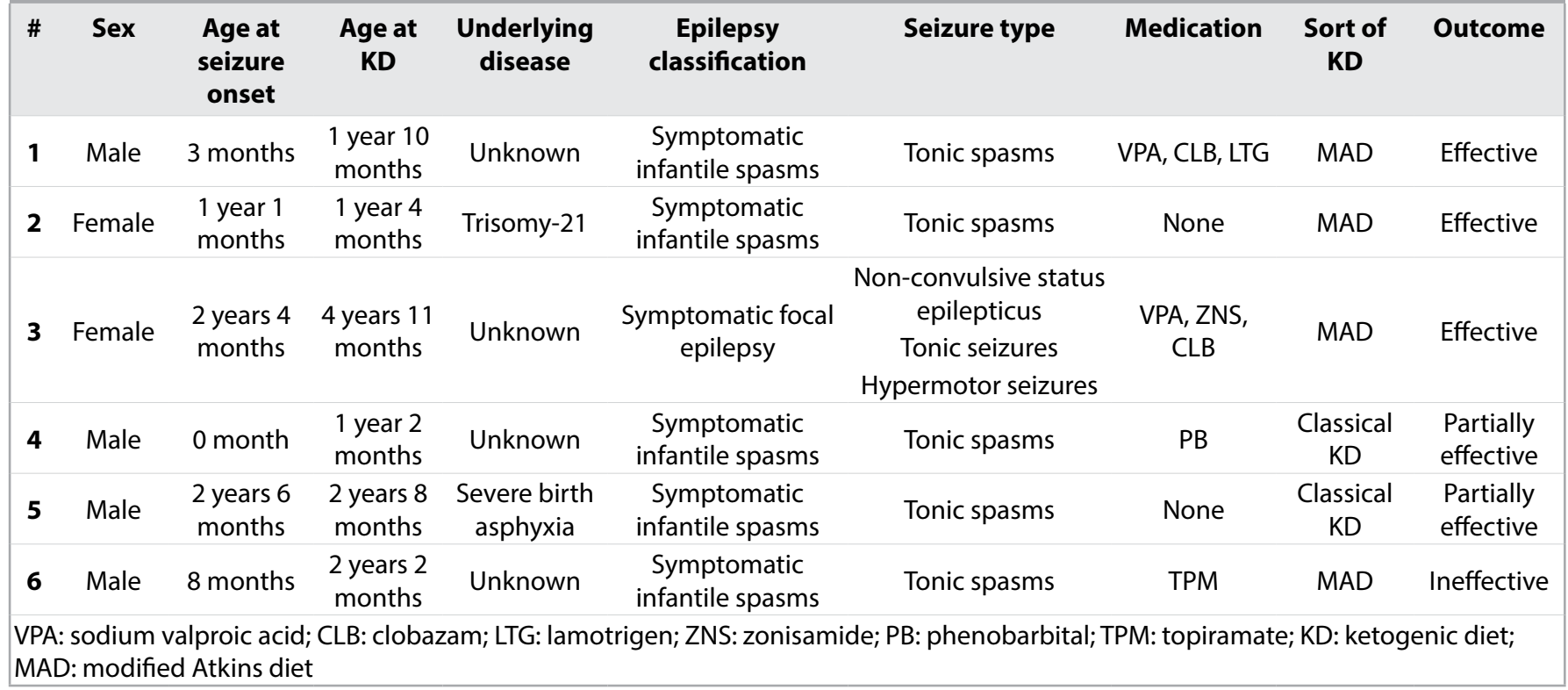

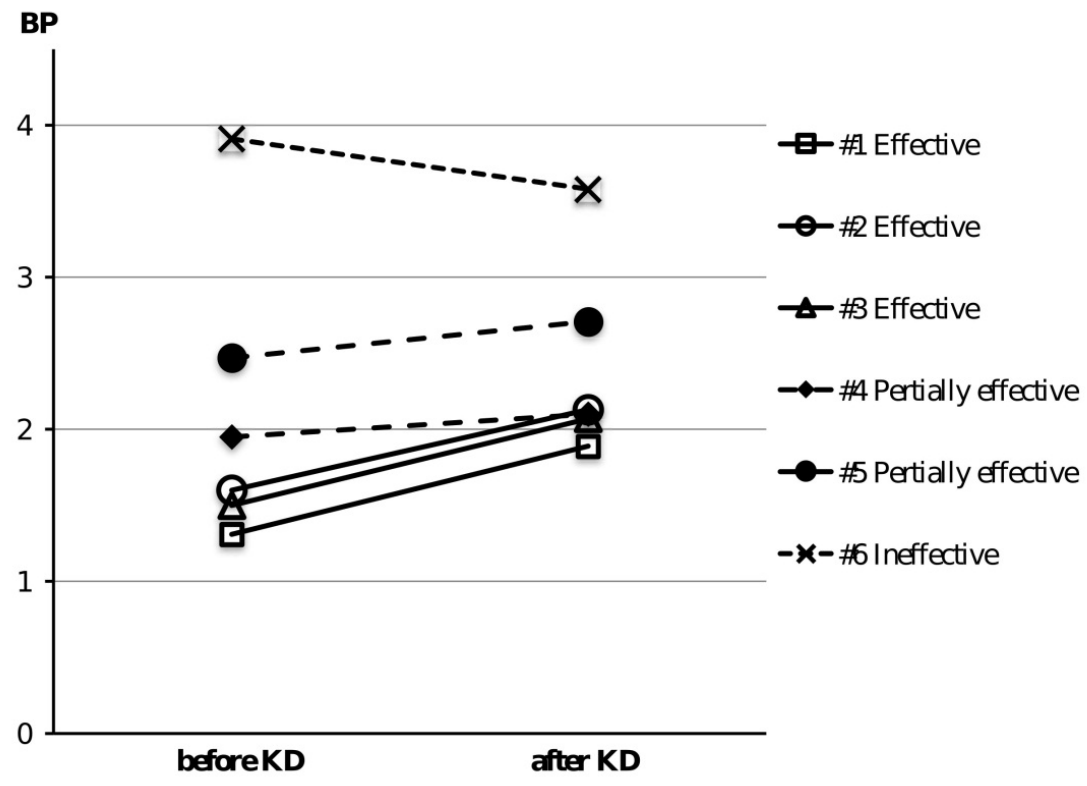

Figure 1. The changes of BP of FMZ during KD in all patients. In KD-effective patients (Case \#1, 2 and 3) increased BP of FMZ. Case \#6 who worsened despite the induction of KD decreased BP of FMZ.

a decrease in the BP area was detected in the bilateral fronto-temporo-parietal lobe; this indicated that a focal lesion may be the cause of his seizures. Conversely, in FIGURE 4, the seizures continued despite two months of KD. His modified hypsarrhythmia was insisted and $\mathrm{BP}$ of FMZ was decreased. A decreased BP area was detected in the right frontal region on PET imaging after $\mathrm{KD}$.

\section{Discussion}

$\mathrm{KD}$ therapy has been clinically useful as a dietary therapy in patients with intractable epilepsy. Its composition of high-fat, lowcarbohydrate foods is believed to alter neurological excitability; however, details of the underlying molecular mechanisms remain unknown. Several proposed mechanisms for the effect of $\mathrm{KD}$ have been recently described [11-14]. The first theory evaluated the role production of ketones, such as $\mathrm{BHB}, \mathrm{ACA}$, and acetone. Ketones have been shown to possess anticonvulsant properties in vivo, but there has been no evidence that they directly 
beforeKD

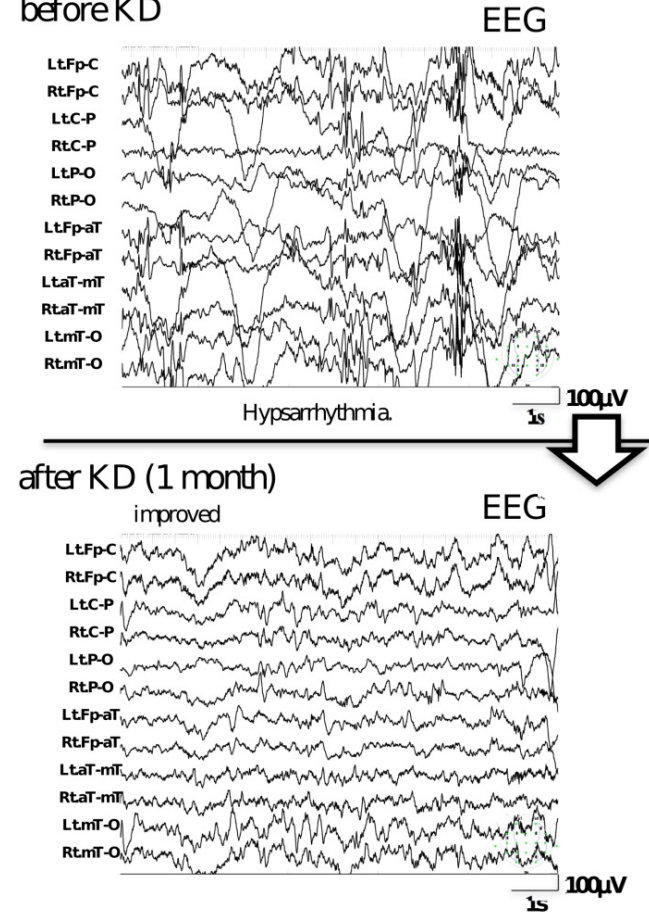

FMZ BP image

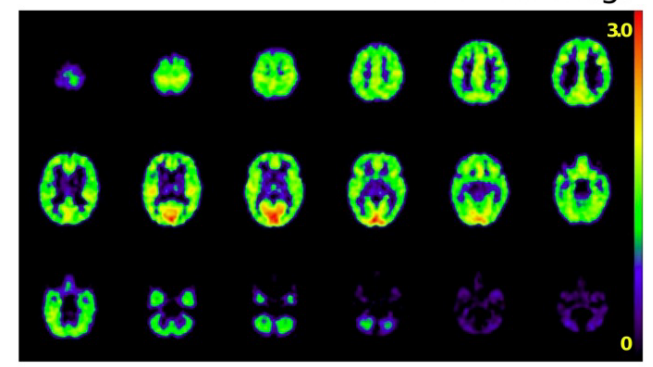

men value of $B P$ in thecerebral cortex $=1.31$

Figure 2. Representative case of KD-effective patient (case \#1) who had experienced refractory epilepsy while being treated with several AEDs (VPA, CLB and LTG). The BP of FMZ was increased, and there was no abnormal BP region in the parametric image at one month after KD.

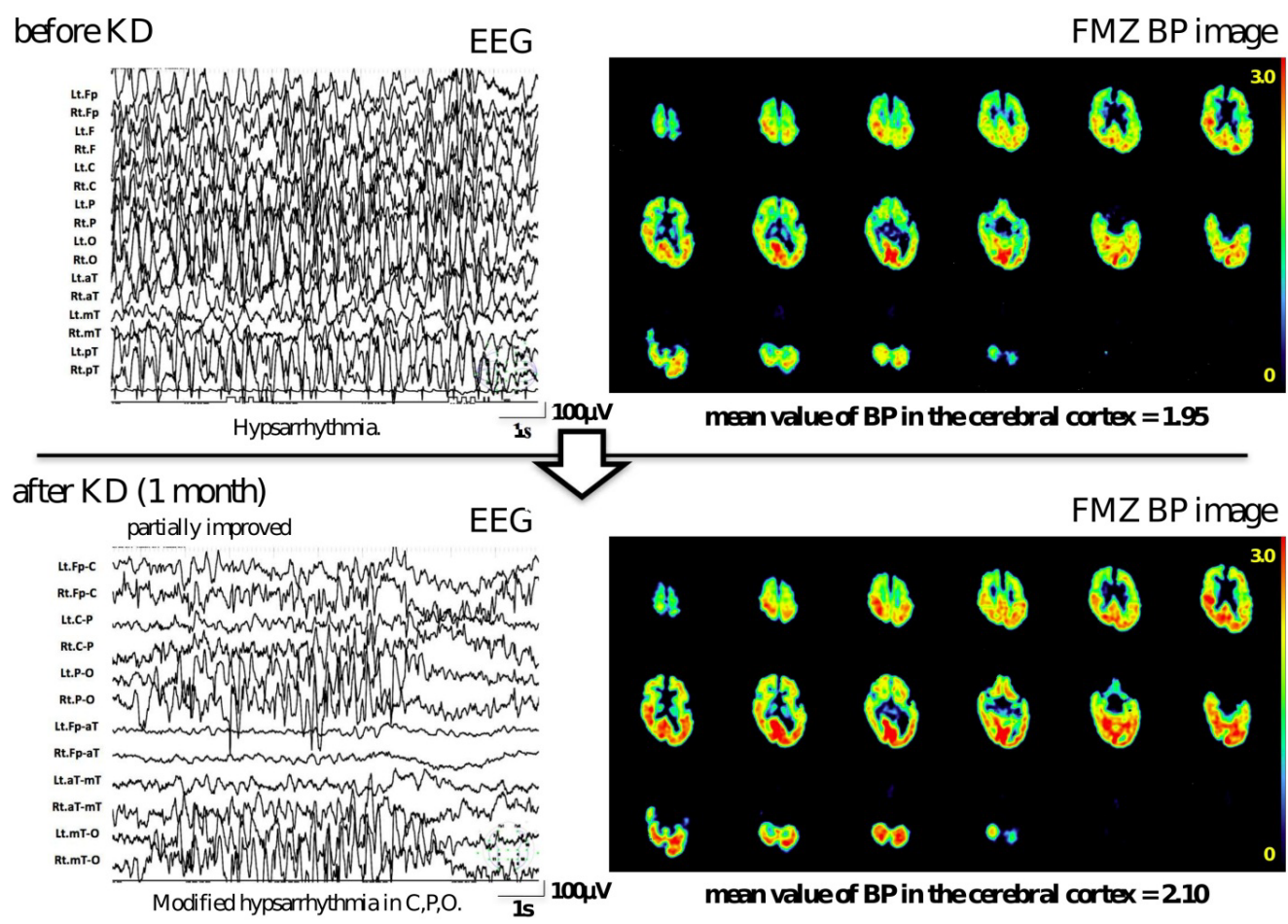

Figure 3. Representative case of KD-partially effective patient (case \#4). The hypsarrhythmia that was found on EEG before KD had partially improved to a modified hypsarrhythmia after KD. The BP of FMZ in the cerebral cortex showed no change and a decrease in the BP area was detected in the bilateral fronto-temporo-parietal lobe. 


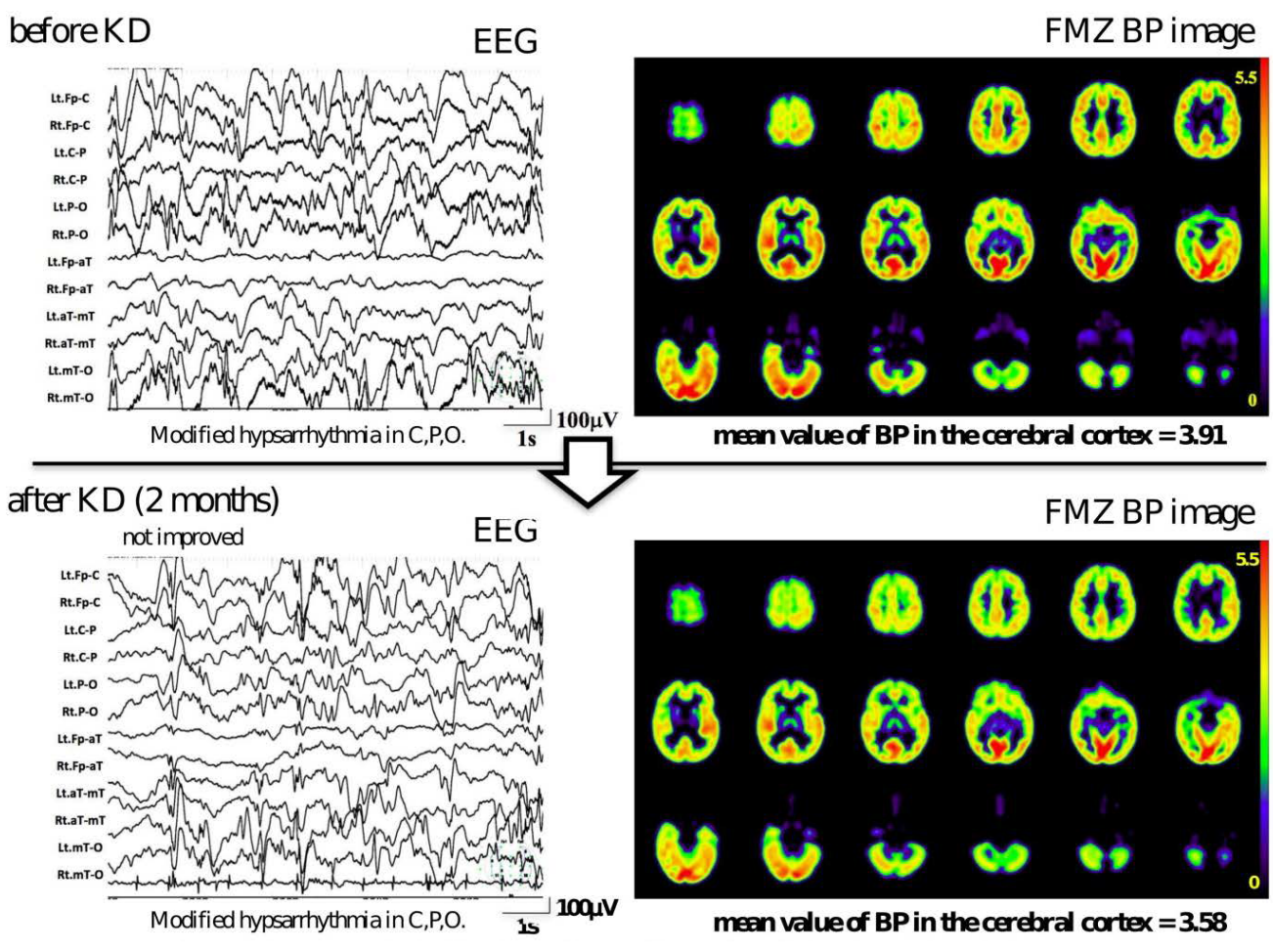

Figure 4. A case of KD-ineffective patient. The seizure continued despite two months of KD. The modified hypsarrhythmia was insisted and BP of FMZ was decreased. A Decreased BP area was detected in the right frontal region in the PET image after KD.

facilitate these effects. Acetone may activate $\mathrm{K}_{2 \mathrm{p}}$ channels, which hyperpolarize cell membranes. The second theory assessed the role of glucose restriction. The $\mathrm{K}_{\text {ATP }}$ channels may open to hyperpolarize the neurons and glia, and the brain-derived neurotrophic factor and trkB signaling, which can promote hyper-excitability and kindling, would be downregulated. Experiments involving 2-deoxyglucose to inhibit glycolysis have provided further support for the glucose-restriction hypothesis of $\mathrm{KD}$ action. The third possibility examined the role of elevated free fatty acids, particularly polyunsaturated fatty acids (PUFAs). Direct actions on ion channels can inhibit both voltagegated $\mathrm{Na}^{+}$and $\mathrm{Ca}^{2+}$ channels and activate $\mathrm{K}_{2 \mathrm{P}}$ channels and $\mathrm{Na} / \mathrm{K}$-ATPase; indirect actions on neuronal excitability can boost the activity of the uncoupling proteins (UCPs), which would limit the generation of reactive oxygen species (ROS). The indirect actions also can upregulate PPAR $\alpha$ expression, which can diminish the IL-1 $\beta$ involved in neuronal hyper-excitability and seizure generation and can stimulate mitochondrial biogenesis, which can enhance energy reserves and lead to stabilized synaptic function. Another proposed mechanism involved the role of metabolic modifications in neurotransmitter synthesis and release. KD enhances norepinephrine (NE) release, which prevents seizure activity in animal models $[33,34]$. Ketosis would modify the TCA cycle and increase glutamate and GABA synthesis. Mitochondrial biogenesis also enhances GABAergic output.

In this study, we had the precious experience of investigating six infants with medicationintractable epilepsy who had been treated with KD therapy and undergone FMZ-PET imaging before and after the diet therapy. All patients who improved after $\mathrm{KD}$ induction showed an increased $\mathrm{BP}$ of FMZ, indicating the activation of the central BZR- related $\mathrm{GABA}_{\mathrm{A}}$-receptor $\left(G_{\left.A B A_{A}-R\right)}\right)$ function in the brain. In contrast, $\mathrm{KD}$-ineffective patients whose clinical condition worsened demonstrated a decreased BP of FM indicating a decrease in $\mathrm{GABA}_{\mathrm{A}}-\mathrm{R}$ function.

We have surmised that there may be several rationales for the increase in $\mathrm{BP}$ of FMZ after $\mathrm{KD}$. In one hypothesis, $\mathrm{KD}$ enhances the $G_{A B A_{A}}-R$ function. Pumain et al. reported that $\mathrm{KD}$ enhanced the $\mathrm{GABA}_{\mathrm{A}}-\mathrm{R}$ function by upregulating $\mathrm{GABA}_{\mathrm{A}}-\mathrm{R}$ phosphorylation with an increase in the kinase activity of glyceraldehyde3-phosphate dehydrogenase (GAPDH) [35]. 
This evidence supports our hypothesis. Another hypothesis hints at another possibility. In the ketotic state, accelerated consumption of oxaloacetate occurs. Oxaloacetate is then less available as a reactant of the aspartate aminotransferase pathway, which couples with the glutamate-aspartate interchange via transamination to the metabolism of glucose through the TCA cycle. Less glutamate is converted to aspartate. Therefore, more glutamate is available for the synthesis of GABA through glutamic acid decarboxylase [36] which is also increased by KD [37]. Another possibility is that the increased GABA levels induced by $\mathrm{KD}$ enhance the affinity of $\mathrm{GABA}_{\mathrm{A}^{-}}$ Rforbenzodiazepine ligands (GABA shift) [38]. We also found that the interictal periods during the FMZ-PET studies could affect the $\mathrm{GABA}_{\mathrm{A}}-\mathrm{R}$ function. The frequencies of seizures in patients were significantly different between before and after KD although both FMZ-PET studies were performed during the interictal state. Bouvard et al. reported that FMZ- PET showed a seizurerelated short-term plasticity of the BZR system in mesial temporal lobe epilepsy patients; this suggested that the regulatory mechanisms of the neurotransmitter system could be studied with FMZ-PET imaging [39]. However, the changes in BP of FMZ over the diffuse cortex as in our cases were not reported in this paper. Pearl et al. also reported a decreased BP of FMZ on FMZ-PET in seven patients with succinic semialdehyde dehydrogenase deficiency [40].

In addition, it is necessary to consider the influence of the drug used for sedation in the PET study. Since all subjects received sedative treatment before PET imaging with the same method, same dose rate, same injection route, and same timing, the influence was considered equivalent to the brain of the drug. Also, there were patients who were taking benzodiazepine drugs as AEDs (CLX, VPA). We determined that it did not have a major impact in the interpretation of the FMZ-PET images.

In the current study, the small number of subjects was a study limitation. Since the population of intractable infantile epilepsy patients is relatively small, it was not easy to register enrollees in this clinical study. Nonetheless, further investigation is needed.

\section{Conclusion}

In conclusion, we performed FMZ-PET studies on patients with intractable epilepsy before and after the initiation of the KD therapy. An increase in BP of FMZ was observed in the parametric BP images of FMZ-PET that were obtained after the initiation of $\mathrm{KD}$, compared with the images obtained before $\mathrm{KD}$ in three patients. However, in one KD-ineffective patient, BP of FMZ decreased during diet therapy. These findings suggested that KD may control seizures by increasing BP of BZR and that FMZ-PET imaging would be useful to assess the efficacy of KD therapy in patients with intractable epilepsy.

\section{Acknowledgement}

We greatly thank Miyuki Takada and Kazumi Sakagami of our hospital, the dietitians for the nutritional education for parents and the design of the KD menu.

\section{REFERENCES}

1. Lee JE, Kim IJ, Cho Kossoff EH, McGrogan JR. Worldwide use of the ketogenic diet. Epilepsia. 46, 280-289 (2005).

2. Vining EP, Freeman JM, Ballaban-Gil K et al. A multicenter study of the efficacy of the ketogenic diet. Arch. Neurol. 55, 1433-1437 (1998).

3. Kossoff EH, McGrogan JR, Bluml RM et al. A modified Atkins diet is effective for the treatment of intractable pediatric epilepsy. Epilepsia. 47, 421-424 (2006).

4. Pires ME, Ilea A, Bourel E et al. Ketogenic diet for infantile spasms refractory to first-line treatments: An open prospective study. Epilepsy. Res. 105, 189-194 (2013).

5. Kossoff EH, Hartman AL. Ketogenic diets: New advances for metabolism-based therapies. Curr. Opin. Neurol. 25, 173-178 (2012).
6. Kumada T, Miyajima T, Kimura $\mathrm{N}$ et al. Modified Atkins diet for the treatment of nonconvulsive status epilepticus in children. $J$. Child. Neurol. 25, 485-489 (2010).

7. Weber S, Molgaard C, Karentaudorf et al. Modified Atkins diet to children and adolescents with medical intractable epilepsy. Seizure. 18, 237-240 (2009).

8. Kossoff EH, Zupec-Kania BA, Rho JM. Ketogenic diets: an update for child neurologists. J. Child. Neurol. 24, 979-988 (2009).

9. Kossoff EH, Dorward JL. The modified Atkins diet. Epilepsia. 49, 37-41 (2008).

10. Kang HC, Lee HS, You SJ et al. Use of a modified Atkins diet in intractable childhood epilepsy. Epilepsia. 48, 182-186 (2007).

11. Kossoff EH, Wang HS. Dietary therapies for epilepsy. Biomed. J. 36, 2-8 (2013).
12. Danial NN, Hartman AL, Stafstrom CE et al. How does the ketogenic diet work? Four potential mechanisms. J. Child. Neurol. 28, 1027-1033 (2013).

13. Masino SA, Rho JM. Mechanisms of ketogenic diet action. Jasper's Basic Mechanisms of the Epilepsies. Bethesda (MD). Natl. Center. Biotechnol. Inf. 1-28 (2012).

14. Bough KJ, Rho JM. Anticonvulsant mechanisms of the ketogenic diet. Epilepsia. 48, 43-58 (2007).

15. Kim MS, Hu HH, Aggabao PC et al. Presence of brown adipose tissue in an adolescent with severe primary hypothyroidism. J. Clin. Endocrinol. Metab. 99, 1686-90 (2014).

16. Petrylak DP. Current state of castration-resistant prostate cancer. Am. J. Manag. Care. 19, 358365 (2013). 
17. Yankam Niwa J, Gray KR, Costes $\mathrm{N}$ et al. Advanced $\left[{ }^{18} \mathrm{~F}\right]$ FDG and $\left[{ }^{11} \mathrm{C}\right]$ Flumazenil PET analysis for individual outcome prediction after temporal lobe epilepsy surgery for hippocampal sclerosis. Neuroimage. Clin. 7, 122-131 (2015).

18. Kumar A, Chugani HT. The role of radionuclide imaging in epilepsy. Part 1: Sporadic temporal and extratemporal lobe epilepsy. J. Nucl. Med. 54, 1775-1781 (2013).

19. Kumar A, Chugani HT. The role of radionuclide imaging in epilepsy. Part 2: Epilepsy syndromes. J. Nucl. Med. 54, 1924-1930 (2013)

20. Frankle WG, Cho RY, Mason NS et al. $\left[{ }^{11} \mathrm{C}\right]$ flumazenil binding is increased in a dosedependent manner with tiagabine-induced elevations in GABA levels. PLoS. One. 7, e32443 (2012).

21. Yamauchi H, Nishii R, Higashi T et al. Selective neuronal damage and Wisconsin card sorting test performance in atherosclerotic occlusive disease of the major cerebral artery. J. Neurol. Neurosurg. Psychiatry. 82, 150-156 (2011).

22. Yamauchi H, Nishii R, Higashi $T$ et al. Silent cortical neuronal damage in atherosclerotic disease of the major cerebral arteries. J. Cereb. Blood. Flow. Metab. 31, 953-961, (2011).

23. Yamauchi $\mathrm{H}$, Nishii $\mathrm{R}$, Higashi $\mathrm{T}$ et al. Hemodynamic compromise as a cause of internal border-zone infarction and cortical neuronal damage in atherosclerotic middle cerebral artery disease. Stroke. 40, 3730-3735 (2009).

24. Yamauchi H, Kudoh T, Kishibe $Y$ et al. Selective neuronal damage and chronic hemodynamic cerebral ischemia. Ann. Neurol. 61, 454-465 (2007).

25. Schmall BCP, Bigler RE, Zanzonico PB et al. Synthesis and quality assurance of $\left[{ }^{11} \mathrm{C}\right]$ alpha-aminoisobutyric acid (AIB), a potential radiotracer for imaging and amino acid transport studies in normal and malignant tissues. Int. J. Nucl. Med. Biol. 11, 209-214 (1984).

26. Kumada T, Nishii R, Higashi $\mathrm{T}$ et al. Ketogenic diet may control seizures by increasing the binding potential of the benzodiazepine receptor: a speculation from the $\left[{ }^{11} \mathrm{C}\right]$ flumazenil-PET study. No. To. Hattatsu. 44, 50-54 (2012).

27. Kumada T, Miyajima T, Oda N et al. Efficacy and tolerability of modified Atkins diet in Japanese children with medication-resistant epilepsy. Brain. Dev. 34, 32-38 (2012).

28. Hartman AL, Vining EP. Clinical aspects of the ketogenic diet. Epilepsia. 48, 31-42 (2007).

29. Okazawa H YH, Sugimoto K, Takahashi M et al. Quantitative comparison of the bolus and steady-state methods for measurement of cerebral perfusion and oxygen metabolism: Positron emission tomography study using ${ }^{15} \mathrm{O}$-gas and water. J. Cereb. Blood. Flow. Metab. 21, 793-803 (2001).

30. Yamauchi H, Kudoh T, Kishibe Y et al. Selective neuronal damage and border zone infarction in carotid artery occlusive disease: A ${ }^{11} \mathrm{C}$-flumazenil PET study. J. Nucl. Med. 46, 1973-1979 (2005).

31. Okazawa H, Yamauchi H, Sugimoto K et al. Effects of metabolite correction for arterial input function on quantitative receptor images with ${ }^{11} \mathrm{C}$-flumazenil in clinical positron emission tomography studies. J. Comput. Assist. Tomogr. 28, 428-435 (2004).

32. Logan JFJ, Volkow ND, Wang GJ et al. Distribution volume ratios without blood sampling from graphical analysis of PET data. $J$. Cereb. Blood. Flow. Metab. 16, 830-840 (1996).
33. Martillotti J, Weinshenker D, Liles LC et al. A ketogenic diet and knockout of the norepinephrine transporter both reduce seizure severity in mice. Epilepsy. Res. 68, 207-211 (2006).

34. Szot P, Weinshenker D, Rho JM et al. Norepinephrine is required for the anticonvulsant effect of the ketogenic diet. Brain. Res. Dev. Brain. Res. 129, 211-214 (2001).

35. Pumain R, Ahmed MS, Kurcewicz I et al. Lability of $\mathrm{GABA}_{\mathrm{A}}$ receptor function in human partial epilepsy: Possible relationship to hypometabolism. Epilepsia. 49, 87-90 (2008).

36. Fakih MG, Fetterly G, Egorin MJ et al. A phase I, pharmacokinetic and pharmacodynamic study of two schedules of vorinostat in combination with 5-fluorouracil and leucovorin in patients with refractory solid tumors. Clin. Cancer. Res. 16, 3786-3794 (2010).

37. Freeman J, Veggiotti P, Lanzi G et al. The ketogenic diet: From molecular mechanisms to clinical effects. Epilepsy. Res. 68, 145-180 (2006).

38. Miller LG, Greenblatt DJ, Barnhill JG et al. GABA shift' in vivo: Enhancement of benzodiazepine binding in vivo by modulation of endogenous GABA. Eur. J. Pharmacol. 148, 123-130 (1988).

39. Bouvard S, Costes N, Bonnefoi F et al. Seizurerelated short-term plasticity of benzodiazepine receptors in partial epilepsy: A $\left[{ }^{11} \mathrm{C}\right]$ flumazenilPET study. Brain. 128, 1330-1343 (2005).

40. Pearl PL, Gibson KM, Quezado Z et al. Decreased GABA-A binding on FMZ-PET in succinic semialdehyde dehydrogenase deficiency. Neurology. 73, 423-429 (2009). 